\title{
Testicular Sparing Surgery: is there a Role in Management of Bilateral Orchidectomy?
}

\author{
SS Goonewardene ${ }^{1 *}$, R Persad ${ }^{2}$, D Gillatt ${ }^{3}$, D Bolton ${ }^{4}$ and N Lawrentschuk ${ }^{4}$ \\ ${ }^{1}$ Guys and St Thomas Hospitals, London \\ ${ }^{2}$ North Bristol NHS Trust, UK \\ ${ }^{3}$ Mcquarrie University, Sydney, Australia \\ ${ }^{4}$ The Austin, Melbourne, Australia
}

Received: 05 February, 2016; Accepted: 29 February, 2016; Published: 10 March, 2016

*Corresponding author: SS. Goonewarene, Urology Registrar, Guys and St Thomas Hospitals, St Thomas St, London, SE1 9RT, Tel: 907897; E-mail: ssg7727@yahoo.co.uk

\begin{abstract}
Background: Germ cell tumours are the most common form of testicular tumour in men less than 45 years. Radical inguinal orchidectomy is the gold standard first line of management. Bilateral testicular tumours present an ongoing management problem. However, testicular sparing surgery is not often considered a common pathway of management.

Population: Adult males ( $\geq 18$ years) with bilateral germ cell tumours and testicular sparing surgery. Intervention: Testicular sparing surgery. Outcomes: Primary outcomes: pathology, tumour size, patient age, survival and oncological outcomes. Due to the paucity of literature in this area, all literature was reviewed- level 1-4.

Objectives: To review outcomes from testicular sparing surgery.

Design: A systematic review was conducted of bilateral testicular germ cell tumours and testicular sparing surgery.

Results and limitations: The systematic review elicited 60 papers, 7 of which conformed to the search criteria.

Conclusions: There are a variety of methods to treat bilateral testicular germ cell tumours, from organ sparing surgery to radio and chemotherapy. With limited evidence we can say, there are specific times this procedure is indicated and patient selection is key.
\end{abstract}

Keywords: Bilateral seminoma; Testicular sparing surgery

\section{Introduction}

The incidence of testicular tumours is 6-8 patients per 100,000 populations [1]. Due to facilities of sperm banking and testicular prostheses, the testis is often considered a none vital organ. The gold standard for testicular cancer, whether mono or bilateral is radical inguinal orchidectomy [2]. However, with questions of hormone replacement this is often difficult for the patient. The overall long term survival rates for patients with germ cell tumours is $100 \%$ [2]. The majority of germ cell tumours are seminomas [3]. Bilateral germ cell tumours are incredibly rare. In cases of bilateral orchidectomy for germ cell tumours, we then have to look and see how we can change our management pathways. Suboptimal androgen replacement therapy results in reduced sexual function and the unpleasant experience of hot [4]. More recently, none organ-sparing techniques have also been advocated for the treatment of malignant testicular and stromal tumors [5].

\section{Methods}

A systematic review relating to literature on bilateral germ cell tumours and testicular sparing surgery was conducted. The search strategy aimed to identify all references related to bilateral germ cell tumours AND testicular sparing surgery. Search terms used were as follows: (Bilateral germ cell tumours) AND (testicular sparing surgery). The following databases were screened from 1984 to November 2015:

- $\quad$ CINAHL

- $\quad$ MEDLINE (NHS Evidence)

- Cochrane

- $\quad$ AMed

- $\quad$ EMBASE

- PsychINFO

- $\quad$ SCOPUS

- Web of Science

In addition, searches using Medical Subject Headings (MeSH) and keywords were conducted using Cochrane databases. Two UK-based experts in prostate cancer care were consulted to identify any additional studies. Studies were eligible for inclusion if they reported primary research focusing on germ cell tumours and surgical management. Papers were included if published after 1984 and had to be in English. Studies that did not conform to this were excluded.

Abstracts were independently screened for eligibility by two reviewers and disagreements resolved through discussion or third party opinion. Agreement level was calculated using Cohen's 
Kappa to test the intercoder reliability of this screening process [6]. Cohens' Kappa allows comparison of inter-rater reliability between papers using relative observed agreement. This also takes account of the comparison occurring by chance. The first reviewer agreed all 5 papers to be included, the second, agreed on 5. For this paper, Cohen's kappa was 1 . Figure 1 demonstrates the results of the screening and selection process [7].

Data extraction was piloted by the researcher and amended in consultation with the research team (author and two academic supervisors). Data collected included authors, year and country of publication, study aims, setting, intervention aims, number of participants, study design, intervention components and delivery methods, comparison groups and outcome measures, notes and follow-up questions for the authors. Studies were quality assessed using the PRISMA criteria [7] for randomised controlled trials, Mays et al. [8] for the action research and qualitative studies and the Critical Skills Appraisal programme for cohort studies. This was also applied to randomised controlled trials and qualitative studies. Meta-analyses for the randomised controlled trials was not appropriate as there were only 3 randomised control trials, and the topics being slightly different (with a varying focus of concern). Population: Adult males ( $\geq 18$ years) with bilateral germ cell tumours and testicular sparing surgery. Intervention: testicular sparing surgery. Comparisons: Experimental vs Control, Experimental vs Experimental, Control vs control.

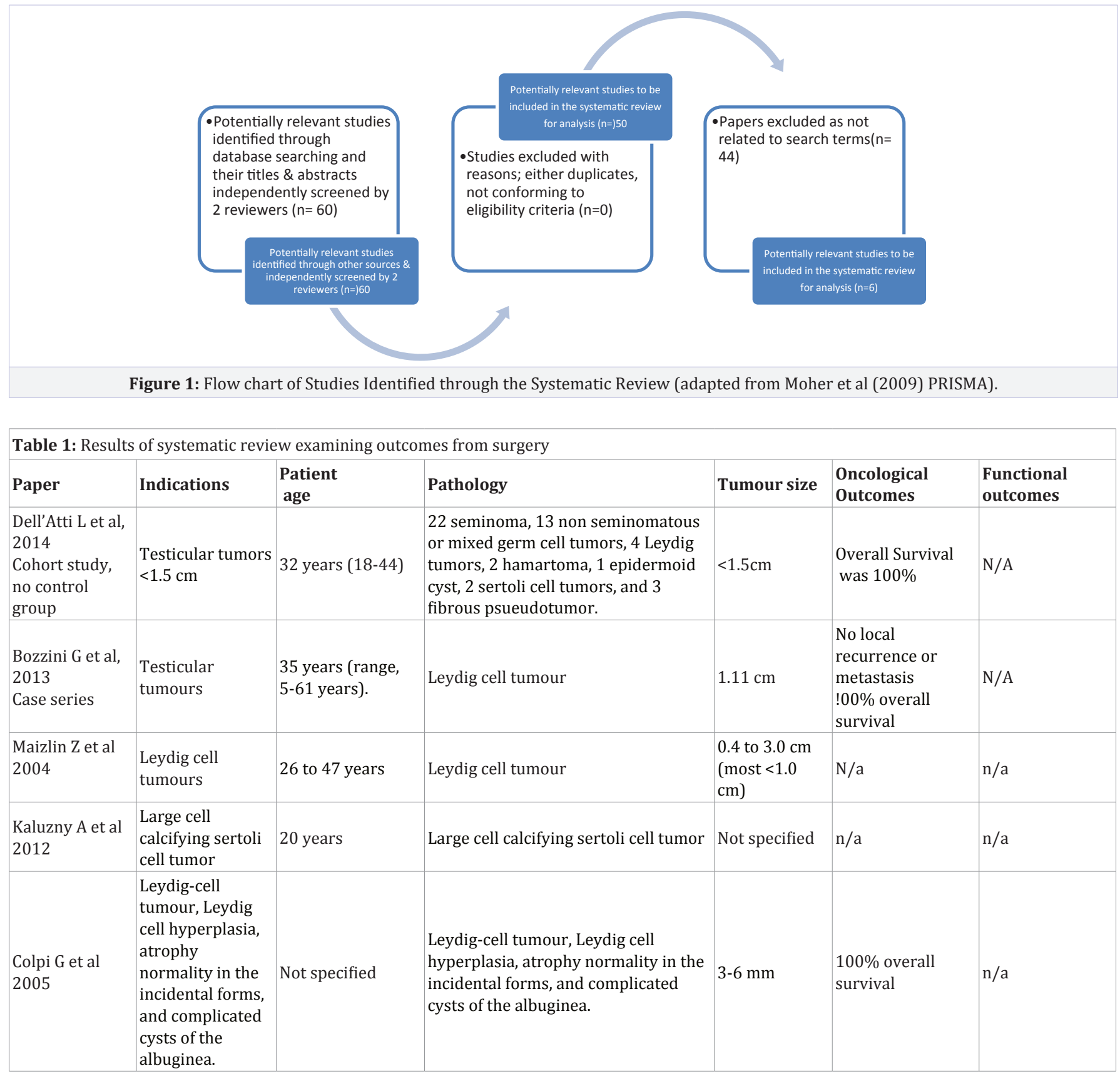


Outcomes: Primary outcomes: Benefits and Harm: Adverse events following treatment. Secondary outcomes: survival outcomes.

\section{Results}

The searches identified 60 papers (Figure 1). However, only 5 mapped to the search terms and eligibility criteria. The current systematic reviews were also examined to gain further knowledge about the subject. 54 papers were excluded due to not conforming to eligibility criteria. Results were presented as per PRISMA criteria [7]. Of the 5 papers left, relevant abstracts were identified and the full papers obtained (all of which were in English), to quality assure against criteria. There was significant heterogeneity within studies, including clinical topic, numbers, outcomes, as a results a narrative review was thought to be best.

For results of the systematic review, please see table 1 . Testicular sparing surgery should be considered for patients presenting with a bilateral tumor on a single testicle [9]. The overall survival rate is $100 \%$ and the low rate of local recurrence (12.8\%) show how effective this procedure is [9]. Long-term follow up suggests that testicle-sparing surgery does not compromise oncologic efficacy in treatment of these tumors [9].

Conservative surgical treatment of Leydig cell tumors using frozen section analysis was also evaluated [10]. Diagnosis after frozen section examination was Leydig cell tumor in 20 of 22 patients [10]. No local recurrences or metastasis were observed [10]. One hundred percent of patients are still alive with a $100 \%$ disease-free survival [10]. This concluded long-term favorable outcomes are seen at follow-up in Leydig cell tumors, even with its potential metastatic behaviour [10]. In these patients, testicle-sparing surgery proved to be a feasible and safe choice and could be regarded as the first line of therapy [10]. Peripheral hypervascularity in a hypoechoic testicular tumor that has little or no internal color Doppler flow should suggest the possibility of a Leydig cell tumor, and consideration should be given to testiclesparing surgery [11].

Large cell calcifying sertoli cell tumor is an exceptionally rare neoplasm originating from sperm cord cells [12]. At presentation, $20 \%$ cases are bilateral and/or multifocal [12]. Consequently, testicle organ-sparing procedure can be attempted, which is especially justified in bilateral lesions [12]. Bilateral tumours have been successfully treated with bilateral testicle-sparing surgery [12].

The increasingly frequent detection of benign testicular lesions, particularly in infertile men, calls for a surgical approach that must be as conservative as possible for the testicular parenchyma [13]. Microsurgery has been successfully used to resect these lesions, whilst preserving fertility within this cohort [13].

However, when we take into account these results, we must bear in mind, there are no control groups.

\section{Conclusion}

This systematic review has quite clearly demonstrated the efficacy of testicular sparing surgery, in cases of bilateral testicular tumour, or in benign cases, however further detailed studies with control groups are required.

\section{Acknowledgements}

For Prof Persad, Prof Gillatt (UK supervisors), Prof Bolton and Prof Lawrentschuk- thank you for your help and support.

\section{References}

1. Steiner H, Holtl L, Maneschg C, Berger AP, Rogatsch H, Bartsch G, et al. Frozen section analysis-guided organ-sparing approach in testicular tumors: Technique, feasibility, and long-term results. Urology. 2003; 62(3): 508-13.

2. Albers P, Albrecht W, Algaba F, Bokemeyer C, Cohn-Cedermark G, Fizazi K, et al. EAU guidelines on testicular cancer: 2011 update. European Urology. 2011; 60(2): 304-19.

3. Gori S, Porrozzi S, Roila F, Gatta G, De Giorgi U, Marangolo M. Germ cell tumours of the testis. Critical Reviews in Oncology/Hematology. 2005; 53(2): 141-64

4. Fossa SD, Opjordsmoen S, Haug E. Androgen replacement and quality of life in patients treated for bilateral testicular cancer. European Journal of Cancer. 1999; 35(8): 1220-5.

5. Heidenreich A, Weissbach L, Holtl W, Albers P, Kliesch S, Kohrmann KU. Organ sparing surgery for malignant germ cell tumor of the testis. Journal of Urology. 2001; 166(6): 2161-5.

6. Cohen J. Weighted kappa: nominal scale agreement with provision for scaled disagreement or partial credit. Psychological bulletin. 1968; $70(4): 213-20$.

7. Moher D, Liberati A, Tetzlaff J, Altman DG. Preferred reporting items for systematic reviews and meta-analyses: The PRISMA statement. Annals of Internal Medicine. 2009; 151(4): 264-9.

8. Mays N, Pope C, Popay J. Systematically reviewing qualitative and quantitative evidence to inform management and policy-making in the health field. Journal of Health Services Research and Policy. 2005; 10(Suppl 1): 6-20.

9. Dell'Atti L, Ippolito C, Fornasari L, Ughi G, Russo GR. Efficacy of ultrasound-guided testicle-sparing surgery for tumors $(<1.5 \mathrm{~cm})$. European Urology, Supplements. 2014; 13(5): 166.

10. Bozzini G, Picozzi S, Gadda F, Colombo R, DeCobelli O, Palou J, et al. Long-term follow-up using testicle-sparing surgery for leydig cell tumor. Clinical Genitourinary Cancer. 2013; 11(3): 321-4.

11. Maizlin ZV, Belenky A, Kunichezky M, Sandbank J, Strauss S. Leydig cell tumors of the testis: Gray scale and color doppler sonographic appearance. Journal of Ultrasound in Medicine. 2004; 23(7): 959-64.

12. Kaluzny A, Matuszewski M, Wojtylak S, Krajka K, Cichy W, Plawski A, et al. Organ-sparing surgery of the bilateral testicular large cell calcifying sertoli cell tumor in patient with atypical Peutz-Jeghers syndrome. International Urology and Nephrology. 2012; 44(4): 1045-8.

13. Colpi GM, Carmignani L, Nerva F, Guido P, Gadda F, Castiglioni F. Testicular-sparing microsurgery for suspected testicular masses. BJU International. 2005; 96(1): 67-9.

14. Johnson LK, Anderson JC, Michael K, Berg J, Quraishi MF. Testicular epidermoid cysts: The "bow tie" pattern of laminated tumors. Journal of Diagnostic Medical Sonography. 2004; 20(3): 162-7. 